\title{
Cryptotanshinone inhibits cellular proliferation of human lung cancer cells through downregulation of IGF-1R/PI3K/Akt signaling pathway
}

\author{
JINGTAO ZHANG $^{1 *}$, GUILAN WEN ${ }^{2 *}$, LONGHUA SUN $^{2,3 *}$, WENXIN YUAN ${ }^{4}$, RAN WANG $^{5}$, \\ QINGHUA ZENG ${ }^{2}$, GUANGYI ZHANG ${ }^{2}$ and BENTONG YU ${ }^{1}$
}

\begin{abstract}
Departments of ${ }^{1}$ Thoracic Surgery and ${ }^{2}$ Respiratory, The First Affiliated Hospital of Nanchang University, Nanchang, Jiangxi 330006; ${ }^{3}$ Jiangxi University of Traditional Chinese Medicine, Nanchang, Jiangxi 330004;

${ }^{4}$ Department of Ultrasonography, The First Affiliated Hospital of Nanchang University, Nanchang, Jiangxi 330006;

${ }^{5}$ Department of Pathology, The First Affiliated Hospital of Sun Yat-sen University, Guangzhou, Guangdong 510080, P.R. China
\end{abstract}

Received January 17, 2018; Accepted July 2, 2018

DOI: $10.3892 /$ or.2018.6638

\begin{abstract}
Lung cancer is one of the most commonly diagnosed malignancies worldwide. Cryptotanshinone (CPT) is a diterpene quinone compound extracted from natural plants and has been reported to have anticancer effects in several cancers including human lung cancer. However, the mechanism by which CPT acts to prevent lung cancer cell growth is largely unknown. In the present study, by using MTT assay, colony formation assay, wound healing and western blotting assays, the effects of CPT on the cell proliferation and migration of human lung cancer cells and the potential cellular signaling mechanisms were investigated. The data demonstrated that CPT exhibited anti-proliferative effects against A549 and H1299 cells. In parallel, the migration of A549 cells was also markedly inhibited by CPT treatment. Further study indicated that CPT not only inhibited the basal phosphorylation level of insulin-like growth factor 1 receptor (IGF-1R) and RAC-alpha serine/threonine-protein kinase (Akt), but also blocked IGF-1 induced IGF-1R and Akt phosphorylation. Finally, it was demonstrated that pretreatment with CPT inhibited IGF-1 induced cell proliferation of A549 and H1299 cells. In
\end{abstract}

Correspondence to: Dr Bentong Yu, Department of Thoracic Surgery, The First Affiliated Hospital of Nanchang University, Nanchang, Jiangxi 330006, P.R. China

E-mail: yubentong@126.com

*Contributed equally

Abbreviations: CPT, cryptotanshinone; EGFR, epidermal growth factor receptor; IGF-1, insulin-like growth factor 1; IGF-1R, insulin-like growth factor 1 receptor; NSCLC, non-small cell lung cancer; PI3K, phosphoinositide 3-kinase; TCM, Traditional Chinese medicine

Key words: cryptotanshinone, lung cancer, insulin-like growth factor 1 receptor, proliferation, migration conclusion, the results of the present study indicated that CPT inhibits the proliferation and migration of lung cancer cells via a mechanism that involves inhibiting the IGF-1R-mediated phosphoinositide 3-kinase/Akt signaling pathway. The data provides evidence that CPT could be developed as a potential therapeutic agent for the treatment of lung cancer.

\section{Introduction}

Lung cancer is considered the second most common cancer and the leading cause of cancer-associated mortalities in both males and females in the United States (1). Based on the differences in clinical behavior and purposes of treatment, lung cancers can be divided into two broad categories, including small cell lung cancer and non-small cell lung cancer (NSCLC). NSCLC is the major type of lung cancer which accounts for $85 \%$ of lung cancer cases $(2,3)$. Despite lung cancer patients can be successfully treated, at least to some extent, by conventional therapeutic strategies including surgery, radiotherapy and chemotherapy, however the majority of patients do not respond well to chemo- and/or radio-therapeutic regimens and the prognosis still remains poor, with a dismal 5-year survival rate of $<15 \%$ (4). Therefore, it is of great importance to discover novel effective therapeutic agents to treat lung cancer.

Unlike synthesized chemo-therapeutic agents which have severe side-effects, traditional Chinese medicines (TCMs), which are made from purely natural compounds, have attracted increasing attention due to less adverse effects for the treatment of cancer (5). A large number of monomer compositions extracted from TCMs have been revealed to exhibit anticancer effects through not only inhibiting cancer cell proliferation, migration and invasion, but also promoting apoptosis and programmed cell death (6). For example, paclitaxel, one of the most successful TCMs extracted from $T$. cuspidata trees, has been revealed to have significant anticancer activity in clinical trials against many types of solid tumors, particularly against metastatic breast cancer and refractory ovarian cancer (7). Notably, in recent years, the active molecular components extracted from the roots of Salvia miltiorrhiza Bunge (also 
named Danshen) have been widely used for treating numerous cardiovascular and endocrine diseases such as coronary artery disease, angina pectoris, hepatitis and menstrual disorders (8). Among them, cryptotanshinone (CPT) has been demonstrated to have bioactivities including anti-inflammatory, anti-oxidative stress and antiplatelet aggregation properties (9). Previously, increasing evidence indicates that CPT appears to exert diverse anticancer properties against numerous human cancer cell lines, such as prostate cancer (10), leukemia (11), gliomas (12), hepatic carcinomas (13), pancreatic (14), breast (15), colorectal (16) and melanoma cancer (17). However, only a few studies have reported the anticancer effects of CPT on lung cancer, and more importantly, the underlying mechanism is largely unknown.

The tyrosine kinase insulin-like growth factor 1 receptor (IGF-1R) plays a pivotal role in the development of cell growth, differentiation and progression of various cancers (18). It has been reported that IGF-1R is overexpressed in many human carcinomas, including breast, prostate and colon cancers $(19,20)$. Conversely, evidence shows that high circulating levels of IGF-1, a ligand of IGF-1R, are associated with increased risk of multiple types of cancers which mainly depends on activation of the downstream signaling of the IGF-1/IGF-1R (21,22). Following activation by IGF-1, the tyrosine kinase activity of IGF-1R phosphorylates the downstream substrates, which in turn results in activation of its downstream signaling pathways, including the phosphatidylinositide 3-kinase/RAC-alpha serine/threonine-protein kinase (PI3K/Akt) signaling cascade (23). Over-activation of PI3K/Akt signaling cascades are largely involved in tumorigenesis and metastasis by extensively phosphorylating apoptotic effector molecules, subsequently leading to cell proliferation and anti-apoptosis (24). Thus, interference with IGF-1R signaling is considered as a potentially effective therapeutic strategy for cancer.

Given the inhibitory effects of CPT on tumorigenesis, the present study aimed to investigate the anticancer effects of CPT on human lung carcinoma A549 and H1299 cells, and explore the potential underlying mechanisms by which it exerts these effects. The results demonstrated that CPT inhibited IGF-1R/PI3K/Akt signaling, which resulted in the inhibition of cell proliferation and migration of lung cancer.

\section{Materials and methods}

Materials. IGF-1 and LY294002 were purchased from Sigma-Aldrich (Merck KGaA, Darmstadt, Germany). CPT was purchased from Tocris Cookson, Inc. (Bristol, UK), Antibodies for western blot analysis, including phospho-Tyr ${ }^{1135 / 1136}$ IGF-1R antibody, IGF-1R antibody, phospho-Ser ${ }^{473}$ Akt antibody, Akt antibody, $\beta$-actin antibody, goat anti-rabbit horseradish peroxidase (HRP)-conjugated were purchased from Cell Signaling Technology, Inc. (Danvers, MA, USA). Reagents used for cell culture including RPMI-1640 and fetal bovine serum (FBS) were purchased from Gibco (Thermo Fisher Scientific, Inc., Waltham, MA, USA).

Cell culture. Human lung adenocarcinoma cells including A549 and H1299 were obtained from the Cell Bank of Chinese Academy of Sciences (Shanghai, China) and cultured according to specifications. The cells were grown in RPMI-1640 medium supplemented with $10 \%$ fetal bovine serum (FBS) and were maintained at $37^{\circ} \mathrm{C}$ in a humidified atmosphere containing $5 \%$ $\mathrm{CO}_{2}$.

Cell proliferation assay. An MTT assay was used to analyze cell proliferation. Briefly, cells in the logarithmic growth phase were seeded in 24-well culture plates at a density of $5 \times 10^{4}$ cells/well and cultured overnight. Cells were treated with different concentrations of CPT for indicated time points (12, 24, 36 and $48 \mathrm{~h})$. Following incubation, $20 \mu \mathrm{l}$ MTT (5 mg/ml, Beyotime Institute of Biotechnology, Haimen, China) was added to each well according to the manufacturer's protocol, and cells were incubated in complete media at $37^{\circ} \mathrm{C}$ for $3 \mathrm{~h}$. The supernatant was removed, then $500 \mu \mathrm{l}$ dimethyl sulfoxide was added to each well of the 24-well plate and gently shaken for $10 \mathrm{~min}$. The absorbance (optical density) at a wavelength of $570 \mathrm{~nm}$ was measured using an enzyme-linked immunosorbent assay reader and used to represent the viability of cells. Results were calculated as percentage of absorbance in control cultures.

Wound healing assay. A total of $2 \times 10^{5}$ cells were seeded into 6 -well plates and cultured to reach $80-90 \%$ confluence. A yellow pipette tip was used to scratch a wound on the midline of each well. The migration of the cells was monitored photographically under a light microscope (Olympus IX83; Olympus Corporation, Tokyo, Japan) and the difference in the area of the wounds was measured before or after application of CPT. The migration rate was expressed as a percentage of the control and was calculated as the proportion of the mean distance between both borderlines to the distance that remained cell free after regrowth.

Colony formation assay. Cells were seeded in a 6-well plate (500 cells/plate) and then treated with CPT for another two weeks. The medium containing CPT was replaced with fresh culture medium every 2-3 days. After two weeks, the supernatants were discarded and the cells were carefully washed with phosphate-buffered saline (PBS). Subsequently, cells were fixed with $4 \%$ paraformaldehyde for $15 \mathrm{~min}$ (at room temperature) and then stained in crystal violet for $20 \mathrm{~min}$ (at room temperature). Images of the colonies were captured by a digital camera.

Transwell assay. Cell migration was assessed using 24-well Transwell cell culture chambers (Corning Incorporated, Corning, NY, USA), according to the manufacturer's protocol. Cells were treated with different concentrations of CPT (5 and $20 \mu \mathrm{M}$ ) for $24 \mathrm{~h}$. Cells were digested with trypsin and resuspended in serum-free medium. Then upper chambers were seeded with $200 \mu$ cells $\left(1 \times 10^{5}\right.$ cells $)$ in serum free medium, and $600 \mu \mathrm{l}$ medium supplemented with $10 \%$ serum was added to the bottom chambers. The chamber was incubated for $24 \mathrm{~h}$ at $37^{\circ} \mathrm{C}$, and after washing twice with PBS, cells were fixed with cold $4 \%$ paraformaldehyde at room temperature and then stained with crystal violate for $15 \mathrm{~min}$ at room temperature. Cells on the bottom surface of the membrane were imaged under an inverted microscope and cells in five randomly selected fields were counted and quantified. 
A<smiles>C[C@@H]1COc2c1ccc1c2C(=O)C(=O)c2c-1ccc1ccccc21</smiles>
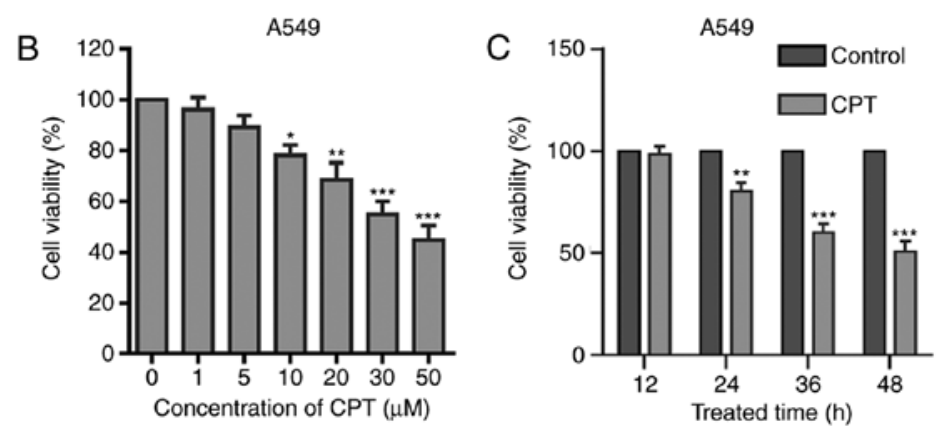

$\mathrm{D}$

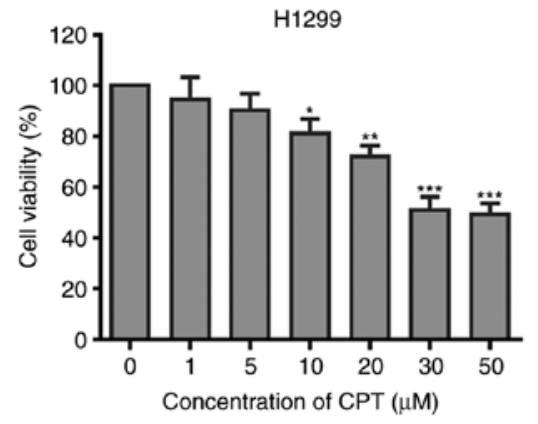

F
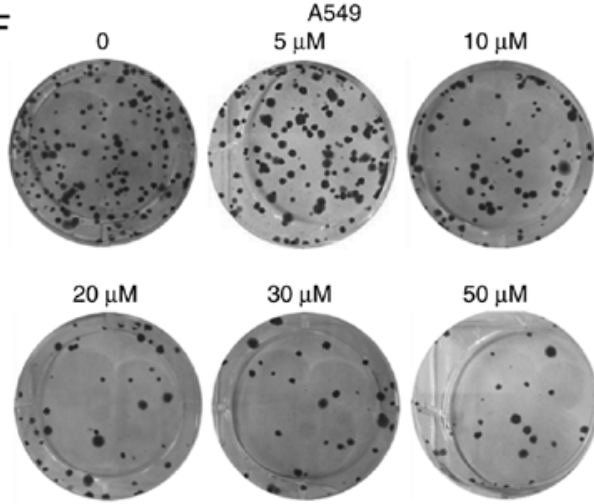
$50 \mu \mathrm{M}$
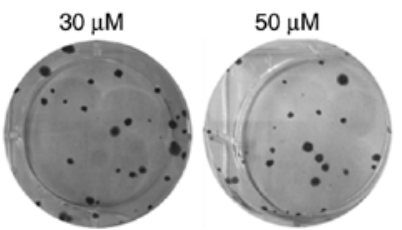
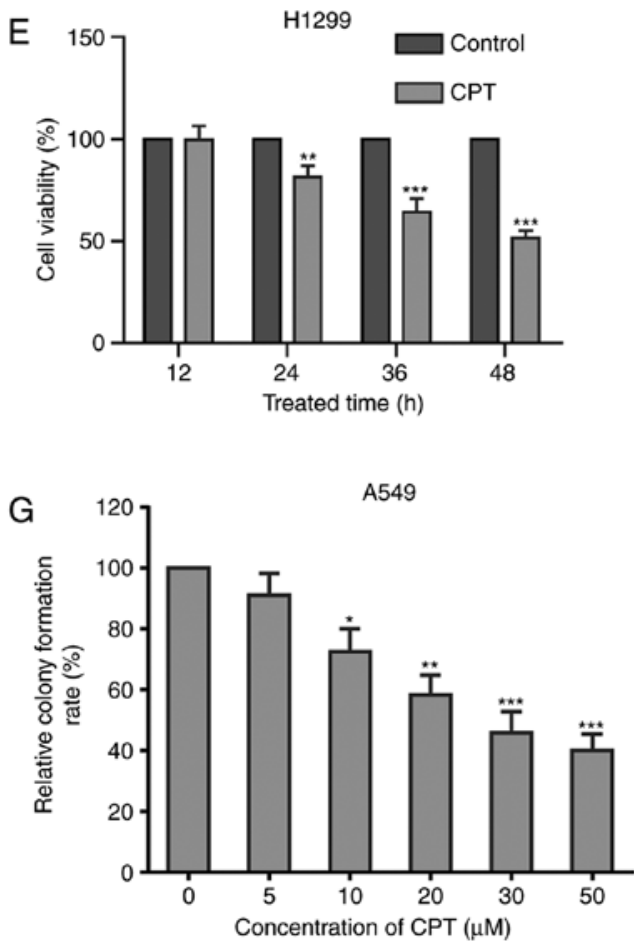

Figure 1. CPT inhibits the cell proliferation of A549 and H1299 cells. (A) The chemical structure of CPT. (B) Effects of different concentrations of CPT on the cell viability of A549 cells. Cells were treated with increasing concentrations of CPT (1, 5, 10, 20, 30 or $50 \mu \mathrm{M})$ for 48 h. Cell viability was measured by the MTT assay. (C) Effects of CPT $(20 \mu \mathrm{M})$ on the viability of A549 cells after 12, 24, 36 and $48 \mathrm{~h}$ treatment. (D) Effects of different concentrations of CPT on the cell viability of H1299 cells. (E) Effects of CPT $(20 \mu \mathrm{M})$ on the viability of H1299 cells after 12, 24, 36 and 48 h treatment. Data are expressed as the mean \pm standard error of the mean SEM (three independent experiments). (F) Cells were pretreated with CPT at the indicated concentrations for two weeks and colonies were photographed. $(\mathrm{G})$ Quantitation of colony formation results. Data was expressed as percentage of control. ${ }^{*} \mathrm{P}<0.05,{ }^{* *} \mathrm{P}<0.01,{ }^{* * * *} \mathrm{P}<0.001$ vs. control group. CPT, crytotanshinone.

Western blotting. Cells were lysed with SDS lysis buffer (Beyotime Institute of Biotechnology, Haimen, China) supplemented with protease inhibitor cocktail. After determination of protein concentration using a modified BCA protein assay (Beyotime Institute of Biotechnology, Haimen, China), equal amounts of protein $(20 \mu \mathrm{g}$ ) were separated by $12 \%$ (for pIGF-1R and IGF-1R, 10\%) SDS-PAGE and transferred to nitrocellulose membranes (EMD Millipore, Billerica, MA, USA). Following blocking with Tris-buffered saline containing 5\% non-fat dry milk and $0.1 \%$ Tween-20 for 1 hat room temperature, membranes were incubated overnight at $4^{\circ} \mathrm{C}$ with anti-phospho-IGF1R (cat. no. 3024; 1:2,000), anti-IGF-1R (cat. no. 9750; 1:2,000), anti-phospho-Akt (cat. no. 9271; 1:2,000), anti-Akt (cat. no. 9272; 1:2,000), anti- $\beta$-actin (cat. no. 4970; 1:4,000), followed by a $2 \mathrm{~h}$ incubation with horseradish peroxidase (HRP)-linked secondary antibodies (cat. no. 7074; 1:20,000) at room temperature. Immunoreactive bands were visualized by enhanced chemiluminescence (Pierce, Thermo Fisher
Scientific, Inc.), and semi-quantification was conducted using Image J 1.47t software (National Institutes of Health, Bethesda, MD) and normalization total protein.

Statistical analysis. Data are presented as the mean \pm standard error of the mean from at least three independent experiments and were analyzed by GraphPad Software Prism 6.0 (GraphPad Software, Inc., La Jolla, CA, USA). Statistical analysis of data was performed using one-way analysis of variance with Bonferroni's multiple comparison tests, or Student's t-test. $\mathrm{P}<0.05$ was considered to indicate a statistically significant difference.

\section{Results}

CPT inhibits the cell proliferation of A549 and H1299 lung cancer cells. As lung cancer is caused by excessive cell growth in the lung and then spreads to nearby tissue in metastasis, the 
A
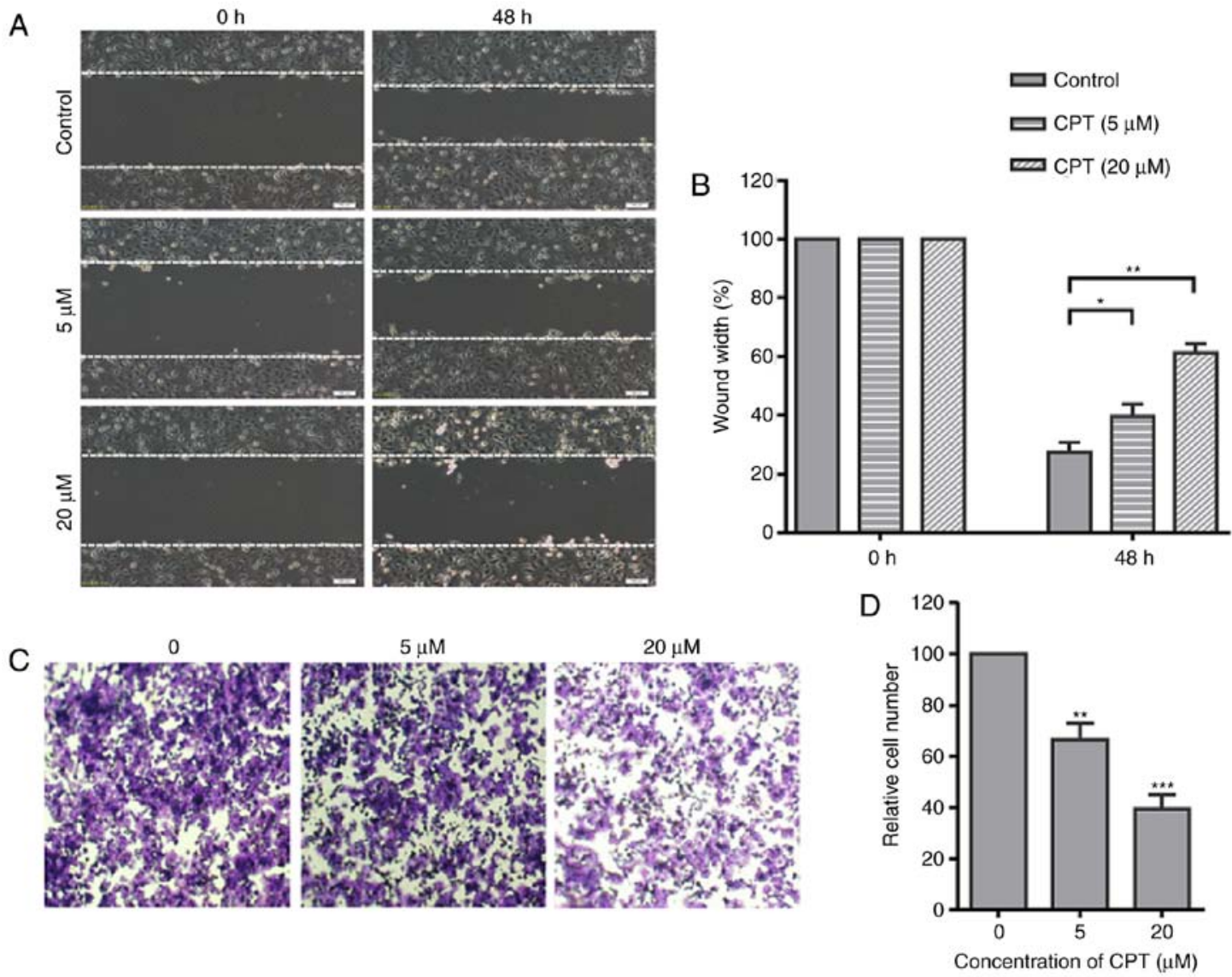

Figure 2. CPT significantly suppresses the migration of A549 cells. (A) Effects of CPT on the migration of A549 cells were measured by wound healing assay. (B) Relative closed-wound distance was calculated by measuring the width of at least four wounds and normalized to control. Data are presented as the mean \pm standard error of the mean. (C and D) Cells were pretreated with or without CPT $(5,20 \mu \mathrm{M})$ for $24 \mathrm{~h}$, harvested, and added to Transwell inserts and incubated for another $24 \mathrm{~h}$. Cell migration was then measured. ${ }^{*} \mathrm{P}<0.05,{ }^{* * *} \mathrm{P}<0.01,{ }^{* * *} \mathrm{P}<0.001$ vs. control group. CPT, crytotanshinone.

present study first investigated the potential role of CPT on cell growth of lung cancer A549 cells. Cells were treated with various concentrations of CPT $(0,1,5,10,20,30$ and $50 \mu \mathrm{M})$ for $48 \mathrm{~h}$. The cell viability was determined by an MTT assay. As presented in Fig. 1, CPT significantly inhibited A549 cell viability in a dose-dependent manner (Fig. 1A and B). Compared with the control group, concentrations of $<5 \mu \mathrm{M}$ had no significant effect, whereas concentrations of $>5 \mu \mathrm{M}$ progressively inhibited the growth of A549 cells (Fig. 1B). Next, the effects of CPT for different treatment times on A549 cell growth were explored, and cells were treated with $20 \mu \mathrm{M}$ CPT for 12, 24, 36 or $48 \mathrm{~h}$. As presented in Fig. 1C, CPT significantly inhibited A549 cell growth, except for at $12 \mathrm{~h}$ incubation, with the strongest effect at $48 \mathrm{~h}$ (Fig. 1C). To confirm whether CPT inhibited cell proliferation of other lung cancer cells, the present study detected the antitumor effect of CPT on H1299 cells, another human non-small cell lung cancer cell line. As presented in Fig. 1D and E, similar results were obtained in H1299 lung cancer cells.

To further explore the effects of CPT on A549 cell growth, a colony formation assay was performed. Consistent with the MTT assay, CPT significantly suppressed the colony formation of A549 cells in a dose-dependent manner (Fig. 1F and G). Taken together, the aforementioned results indicated that CPT exhibits a significant inhibitory effect toward cell growth in lung cancer cells.
CPT significantly suppresses the migration of A549 cells. Migration of cancer cells is a key factor responsible for cancer metastasis, and the present study investigated whether CPT could affect the migration of A549 cells. To investigate the effects of CPT on the migration of A549 cells, a typical scratch wound healing assay was performed to measure the migration ratio. The confluent monolayer was scraped with a sterile yellow micropipette tip to create a scratch wound. After incubation with 5 or $20 \mu \mathrm{M}$ CPT for $48 \mathrm{~h}$, the cells migrated to the denuded zone and then the width of gaps of the wound area were measured. As presented in Fig. 2, CPT significantly inhibited the migration of A549 cells at $48 \mathrm{~h}$ after treatment (Fig. 2A and B). To further confirm this result, a Transwell assay was performed to quantify the migratory ability of A549 cells. The results revealed that CPT induced a dose-dependent inhibition in migration with CPT (Fig. 2C and D). These results indicated that $\mathrm{CPT}$ significantly inhibits the migration of A549 cells, even at a low dose.

CPT inhibits IGF-1R and Akt phosphorylation in A549 cells. The activation of receptor tyrosine kinases such as IGF-1R signaling has been reported to be crucial for lung cancer cell proliferation, survival, invasion and angiogenesis (25). Therefore, we explore whether IGF-1R signaling is involved in the anti-proliferation effect of CPT. Cells were treated with CPT $(20 \mu \mathrm{M})$ for different time points as indicated and 
A
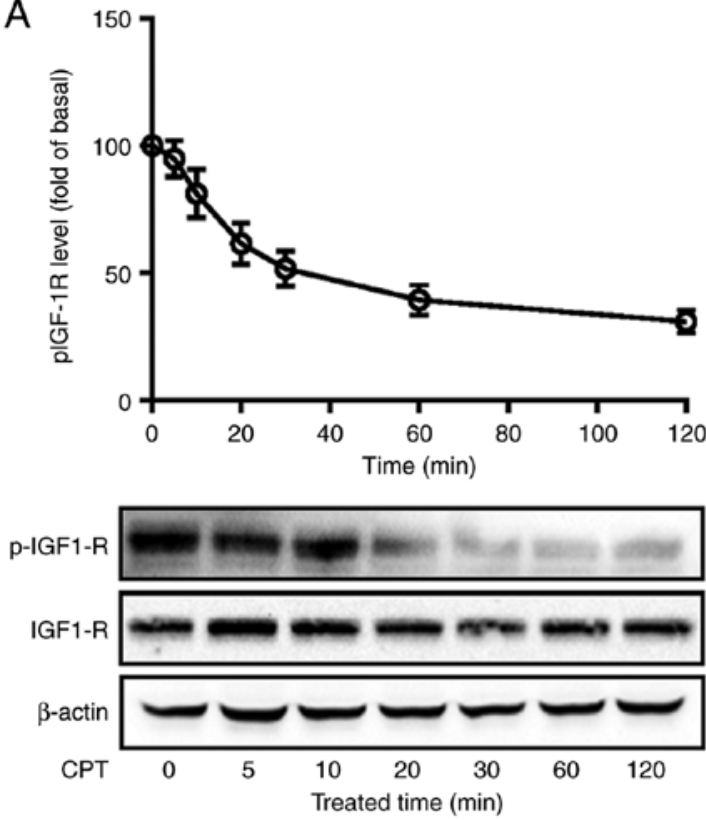

C
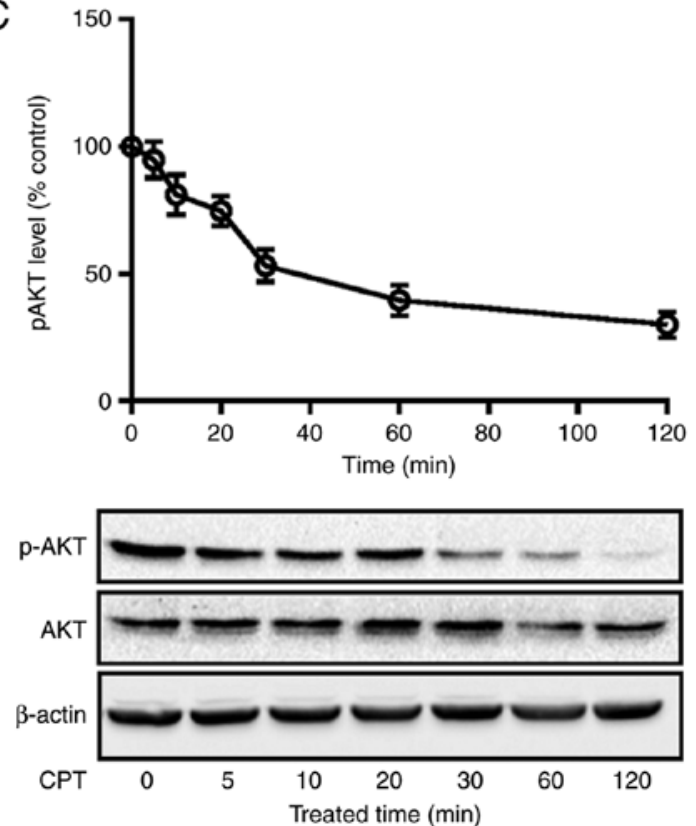

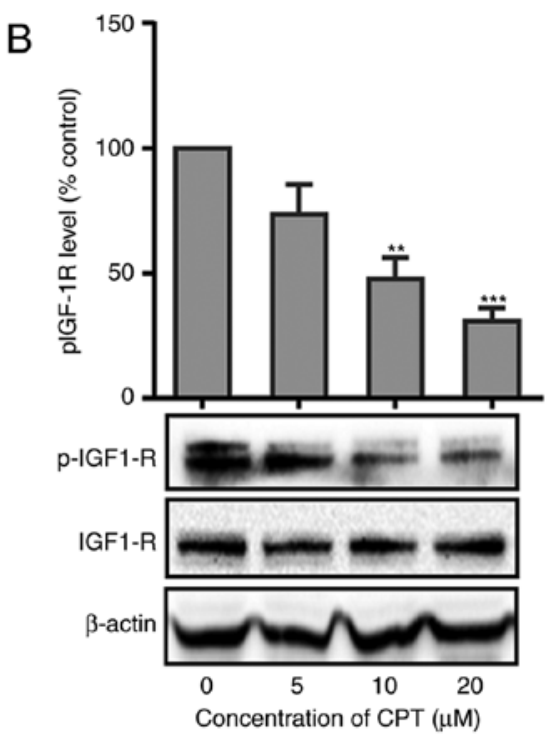

D

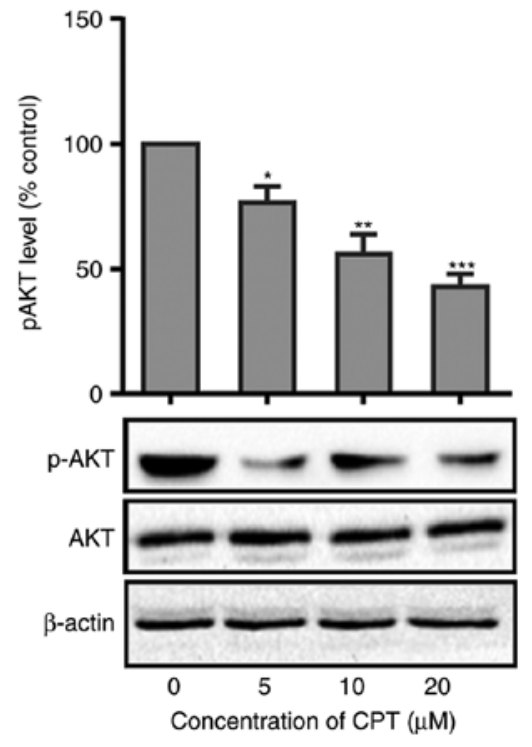

Figure 3. CPT inhibits IGF-1R and Akt phosphorylation in A549 cells. (A) Cells were treated with CPT (20 $\mu \mathrm{M})$ at the indicated times points. IGF-1R phosphorylation was determined by western blotting. (B) Cells were treated with several concentrations of CPT (5, 10 and $20 \mu \mathrm{M})$ for 30 min, then IGF-1R phosphorylation was measured. (C) Cells were treated with CPT $(20 \mu \mathrm{M})$ for different time points as indicated above, and Akt phosphorylation was determined. (D) Cells were treated with different concentrations of CPT $(5,10$ and $20 \mu \mathrm{M})$ for 30 min then Akt phosphorylation was measured. Data represent the mean \pm standard error of the mean of at least three independent experiments. ${ }^{*} \mathrm{P}<0.05,{ }^{* *} \mathrm{P}<0.01,{ }^{* * *} \mathrm{P}<0.001$ vs. control group. CPT, crytotanshinone; IGF-1R, insulin-like growth factor 1 receptor; Akt, RAC-alpha serine/threonine-protein kinase.

IGF-1R phosphorylation was detected by western blotting. As presented in Fig. 3A, CPT caused a rapid decrease in the phosphorylation level of IGF-1R (Fig. 3A). However, it had no effect on the total IGF-1R expression levels. Following this, the phosphorylation level of IGF-1R was detected after cells were treated with different concentrations of CPT $(5,10$ and $20 \mu \mathrm{M})$ for $30 \mathrm{~min}$. As presented in Fig. 3B, IGF-1R phosphorylation was also significantly decreased (Fig. 3B).

The PI3K/Akt signaling pathway, which is an important downstream signaling cascade of IGF-1R, regulates various cellular functions relevant to the growth and progression of lung cancer (26). The present study next measured the effects of CPT on the phosphorylation level of Akt. As presented in Fig. 3C, CPT significantly decreased the phosphorylation level of Akt in the same manner as IGF-1R phosphorylation (Fig. 3C and D). The aforementioned results suggest that CPT can significantly inhibit the IGF-1R/Akt signaling pathway in A549 cells.

CPT inhibits IGF-1-induced Akt phosphorylation in A549 and H1299 cells. It has been demonstrated that high circulating levels of IGF-1 are associated with increased risk of multiple types of cancers including lung cancer. It was then determined whether CPT could inhibit IGF-1-induced activation 

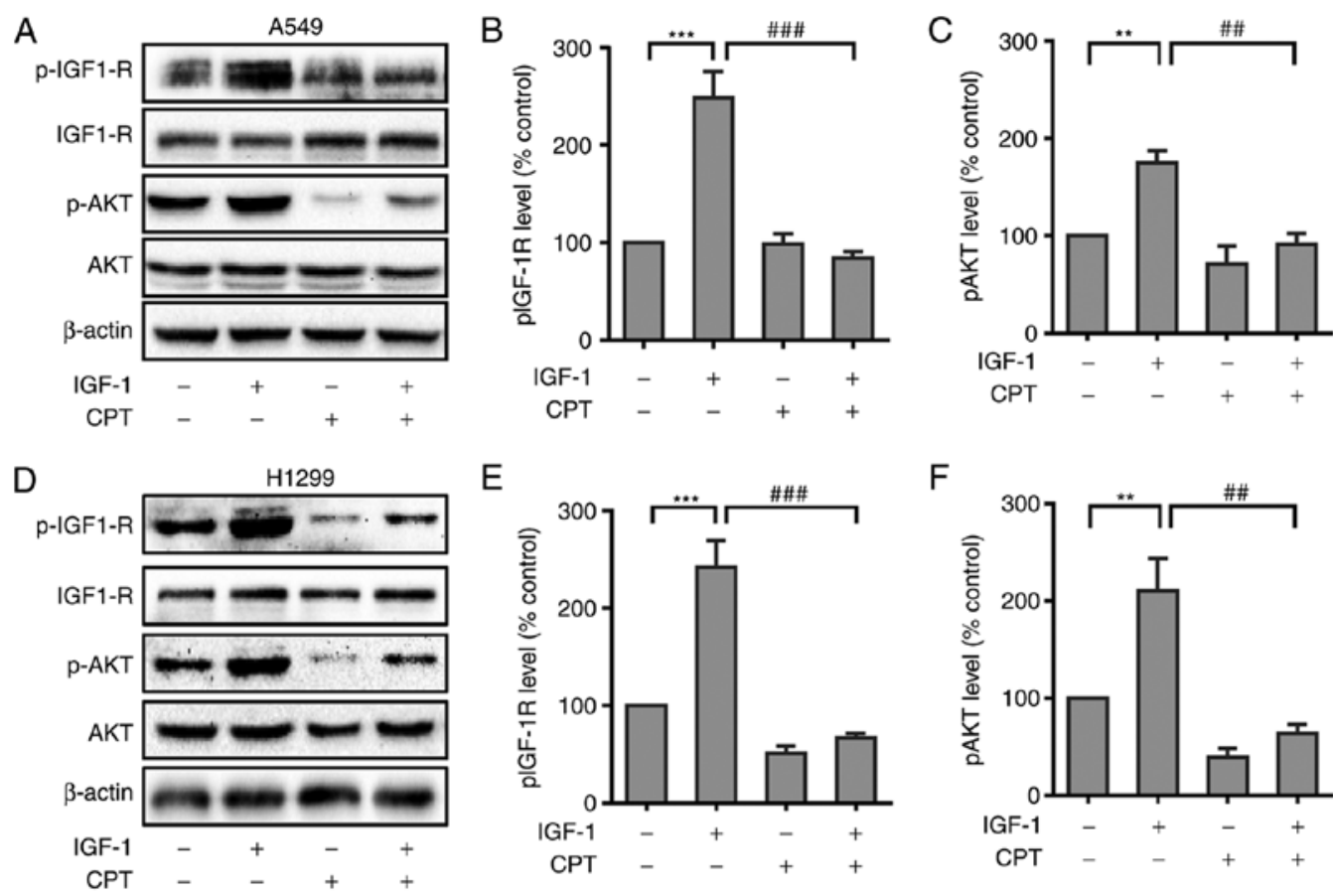

E

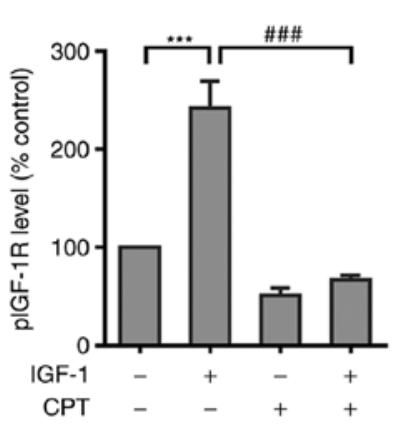

$\mathrm{F}$
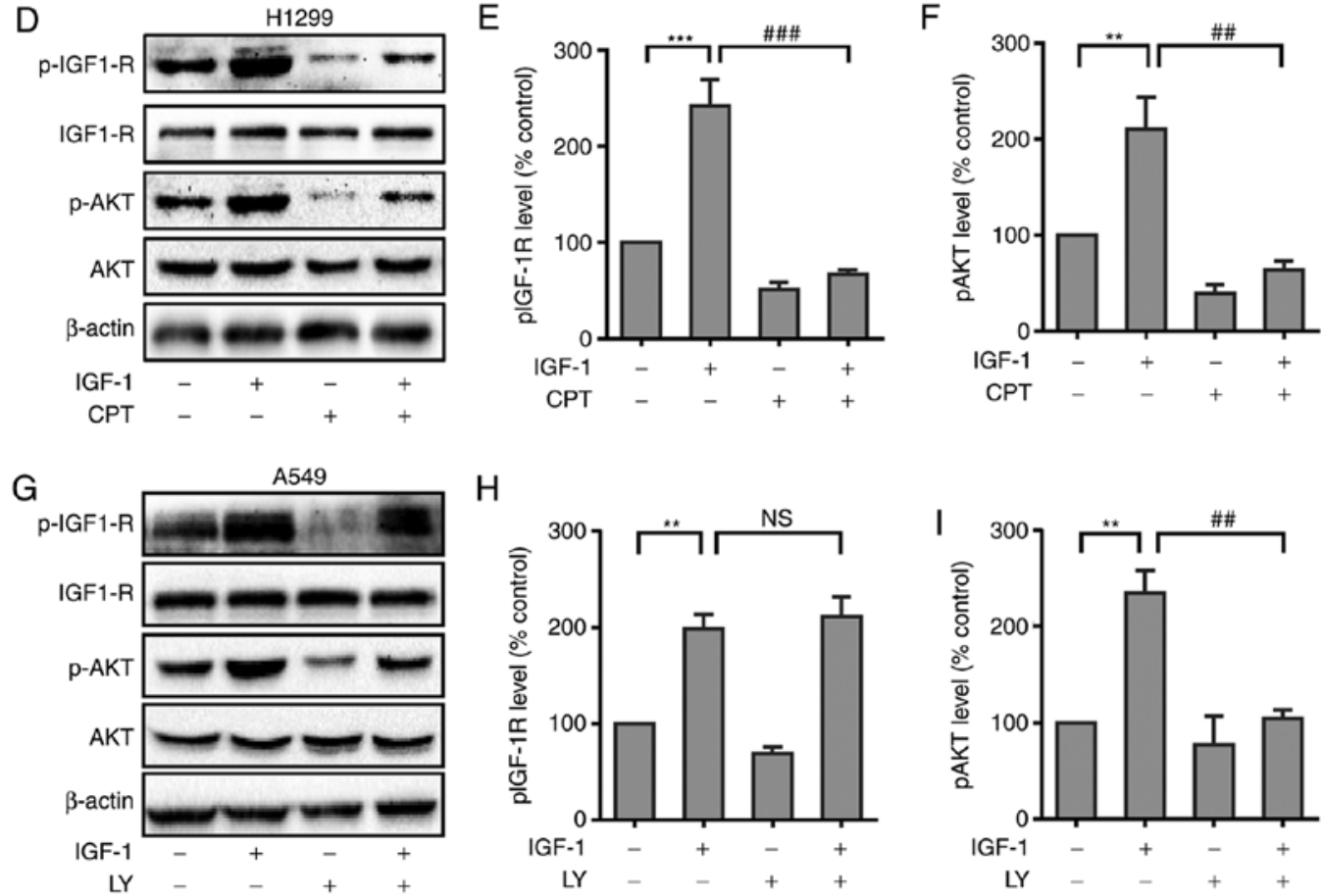

Figure 4. CPT inhibits IGF-1-induced IGF-1R and Akt phosphorylation in A549 and H1299 cells. (A) Effects of CPT on IGF-1-induced IGF-1R and Akt phosphorylation. The relative phosphorylation levels of (B) IGF-1R and (C) Akt in each group of A549 cells were analyzed by densitometry normalized to $\beta$-actin. (D) Effects of CPT on IGF-1-induced IGF-1R and Akt phosphorylation. The relative phosphorylation levels of (E) IGF-1R and (F) Akt in each group of H1299 cells were analyzed by densitometry normalized to $\beta$-actin. (G) Effects of phosphoinositide 3-kinase inhibitor LY on IGF-1-induced IGF-1R and Akt phosphorylation. The relative phosphorylation levels of (H) IGF-1R and (I) Akt in each group were analyzed by densitometry normalized to $\beta$-actin. Data represent the mean \pm standard error of the mean from at least three independent experiments. ${ }^{* *} \mathrm{P}<0.01,{ }^{* * * *} \mathrm{P}<0.001$ vs. control group; ${ }^{\# \#} \mathrm{P}<0.01,{ }^{\# \# \#} \mathrm{P}<0.001$ vs. IGF-1-treated group. LY, LY294002; CPT, crytotanshinone; IGF-1R, insulin-like growth factor 1 receptor; Akt, RAC-alpha serine/threonine-protein kinase; p, phosphorylated.

of IGF-1R signaling. As presented in Fig. 4A, compared with control group, the phosphorylation level of IGF-1R was greatly enhanced in A549 cells treated with IGF-1. As expected, pretreatment of A549 cells with CPT significantly inhibited IGF-1 induced phosphorylation of IGF-1R (Fig. 4A and B). Furthermore, CPT also inhibited the IGF-1-induced phosphorylation of Akt (Fig. 4A and C). Additionally, similar results were observed in H1299 cells (Fig. 4D-F), suggesting CPT can significantly inhibit IGF-1R and Akt signaling cascades in lung cancer cells.

To confirm whether Akt phosphorylation was downstream of IGF-1R in lung cancer cells, the present study next evaluated the effects of the PI3K selective inhibitor LY294002 on IGF-1-induced Akt phosphorylation in A549 cells. Cells were pretreated with LY294002 $(20 \mu \mathrm{M})$ for $30 \mathrm{~min}$ and then stimulated with IGF-1. It was demonstrated that LY294002 did not have a significant effect on IGF-1R phosphorylation (Fig. 4G and $\mathrm{H}$ ), however blocked IGF-1-induced Akt phosphorylation (Fig. 4G and I), suggesting Akt acts as a downstream factor of PI3K signaling in A549 cells. This suggested that PI3K acts as a downstream signaling molecule of IGF-1R.

CPT inhibits IGF-1-induced A549 and H1299 cell proliferation. Given the ability of CPT to significantly inhibit proliferation and IGF-1R/PI3K/Akt phosphorylation in A549 cells, it was hypothesized that the inhibitory effect of CPT on cell proliferation was mediated by the IGF-1R/PI3K/Akt pathway. To verify this hypothesis, the present study determined whether CPT was able to inhibit IGF-1-induced cell proliferation of A549 cells or not. The cells were treated with IGF-1 $(20 \mathrm{ng} / \mathrm{ml})$ alone or in combination with CPT $(20 \mu \mathrm{M})$ for $48 \mathrm{~h}$ and then cell viability was measured by MTT assay. As presented in Fig. 5A, IGF-1 treatment alone significantly increased cell proliferation of A549 cells, which is associated with the activated IGF-1R/PI3K/Akt pathway. However, this 
A

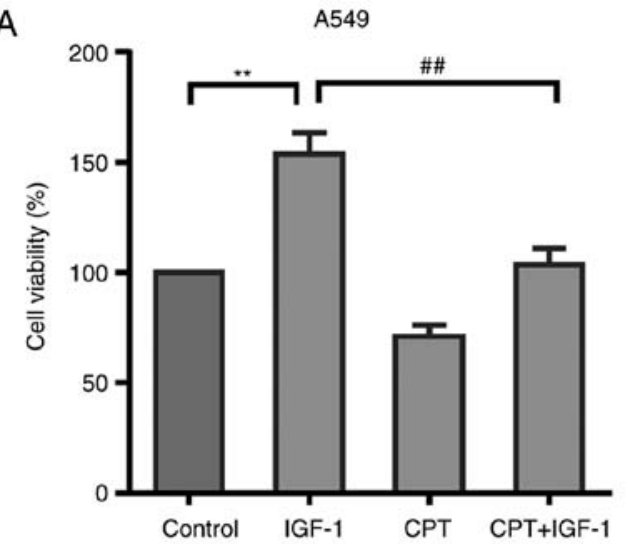

C
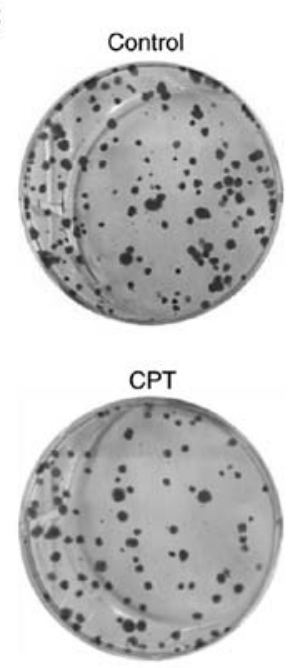

A549
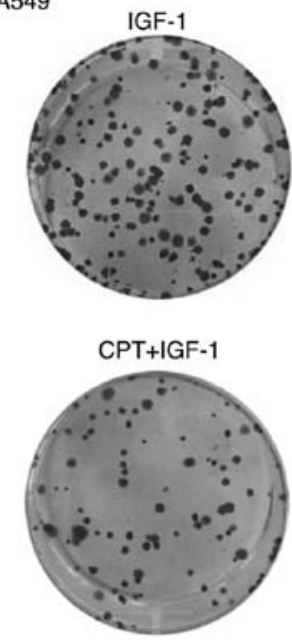

B
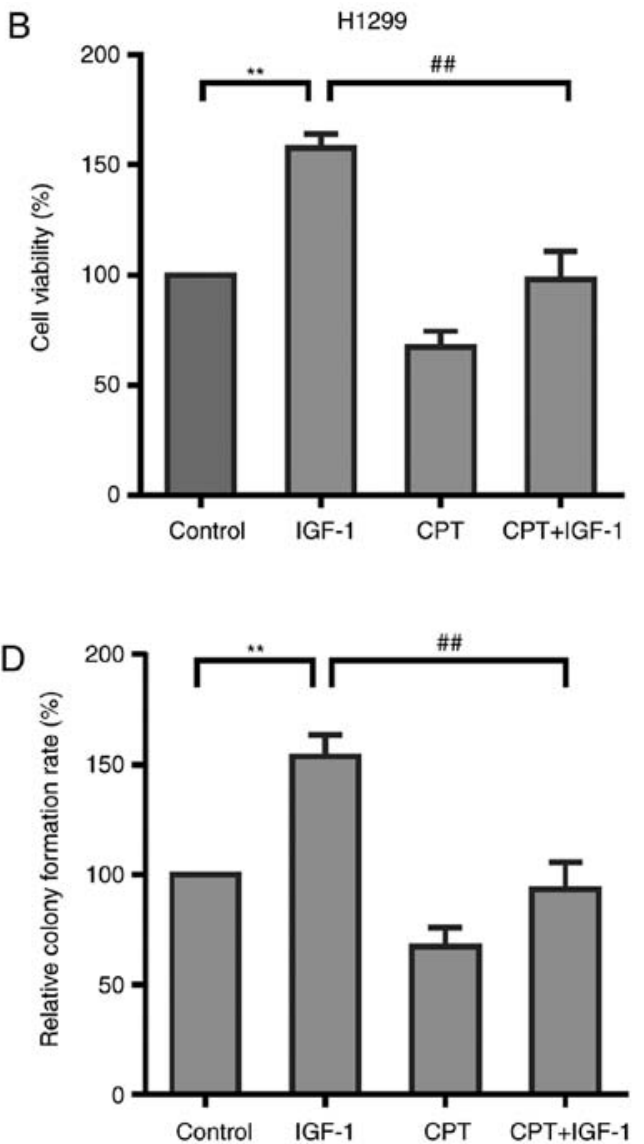

Figure 5. CPT inhibits IGF-1-induced A549 and H1299 cell proliferation. (A) A549 and (B) H1299 cells were treated with IGF-1 (20 ng/ml) alone or in combination with CPT $(20 \mu \mathrm{M})$ for $48 \mathrm{~h}$, then cell viability was measured by MTT assay. (C) Effects of CPT on IGF-1-induced colony formation capacity of A549 cells. (D) Quantitation of colony formation results as shown in (B). ${ }^{* *} \mathrm{P}<0.01$ vs. control group; ${ }^{\# \#} \mathrm{P}<0.01$ vs. IGF-1-treated group. CPT, crytotanshinone; IGF-1R, insulin-like growth factor 1 receptor.

IGF-1-enhanced proliferation was significantly suppressed by CPT pretreatment (Fig. 5A). CPT was also able to inhibit IGF-1-induced cell proliferation in H1299 cells (Fig. 5B). The result was verified by a colony formation assay. As expected, CPT significantly inhibited IGF-1 induced colony formation capacity (Fig. 5B and C). Taken together, these results suggest that CPT may suppress A549 cell proliferation via inhibiting IGF-1R/PI3K/Akt pathway.

\section{Discussion}

Lung cancer is a leading cause of cancer-associated mortality worldwide. An increasing number of people, particularly adult men, suffer fatalities from lung cancer than any other type of cancers. Due to the high incidence and mortality of lung cancer, development of novel therapeutic agents is urgently needed. The present study investigated the anticancer effects of CPT on human lung carcinoma A549 and H1299 cells and explored the potential cell signaling mechanism. The results indicated that CPT not only inhibited the proliferation and migration, but also suppressed the IGF-1 induced proliferation of A549 and H1299 cells. Furthermore, the anticancer effect of CPT may be attributable to inhibition of the IGF-1R/PI3K/Akt signaling pathway.
The traditional Chinese medicine Salvia miltiorrhiza Bunge has been used as an anticancer agent for many years. To date, several effective compounds including tanshinones I, IIA and IIB, dihydrotanshinone and CPT which are extracted from the roots of Salvia miltiorrhiza Bunge have been demonstrated to possess an anticancer effect in multiple cancer types. However, most studies focused on the anti-oxidant, antiinflammatory and anticancer effects (7) of tanshinone I and IIA (8). In recent years, more and more studies indicated that $\mathrm{CPT}$ also exerts anticancer effects, mainly by inhibiting cancer cell proliferation and migration and inducing cancer cell apoptosis in multiple cancer types. A recent study showed that CPT inhibits the cell growth of lung cancer cells (27). However, the underlying molecular mechanisms of such an effect are not fully understood. The present study investigated the potential anticancer effect of CPT and revealed the underlying mechanisms: CPT suppressed lung cancer cell proliferation and migration through inhibiting IGF-1R/PI3K/Akt signaling cascades.

It has been reported that IGF-1R is aberrantly expressed in many types of cancers. Both increased IGF-1R expression levels and high circulating levels of its ligand IGF-1 are associated with higher risk of developing various malignant tumors which mainly depends on activation of 
the downstream cascade of IGF-1/IGF-1R signaling $(21,28)$. Notably, an increasing number of studies have demonstrated that IGF-1R-mediated downstream signaling pathways are overactivated during the early stages of carcinogenesis in various malignant tumors including lung cancer, and a role for IGF-1R signaling has been demonstrated not only in primary tumor formation but also in progression to more aggressive lung adenocarcinoma (13). Based on the aforementioned findings, IGF-1R is considered as an important target for the prevention of lung cancer and various other types of cancer formation. The present study demonstrated that CPT inhibited IGF-1R phosphorylation in both A549 and H1299 lung cancer cells. CPT also inhibited IGF-1-induced IGF-1R phosphorylation and cell proliferation of these cells. These results suggested that CPT may suppress A549 and H1299 cell proliferation through IGF-1R signaling. However, the underlying mechanism by which CPT acts to suppress IGF-1R phosphorylation requires further investigation in the future. Since other growth factor receptors like epidermal growth factor receptor (EGFR) are overexpressed in 40-80\% of NSCLCs (28), whether CPT can also inhibit EGFR signaling remains elusive.

The PI3K/Akt pathway integrates signals from external cellular stimuli such as growth factors and cytokines to regulate essential cellular functions and is aberrantly activated in various types of human cancers (24). Accumulating studies indicate that the PI3K/Akt pathway plays a critical role in regulating various cellular functions such as proliferation, survival, invasiveness and metastasis of cancer cells $(29,30)$. Moreover, clinical studies also demonstrated that activation of the $\mathrm{PI} 3 \mathrm{~K} / \mathrm{Akt}$ pathway is highly correlated with tumor progression and reduced patient survival (31). Thus, the PI3K/Akt pathway is considered a promising target for anticancer pharmacological agents (32). The present study demonstrated that CPT not only blocked basal Akt phosphorylation but also inhibited IGF-1 induced Akt activation. Together with the results that CPT inhibited both basal level and IGF-1-induced A549 and H1299 cell proliferation, the data suggest the involvement of the PI3K/Akt pathway in CPT-induced inhibition of cell proliferation.

Deregulation of apoptosis is the hallmark of all cancer cells, and the induction of apoptosis has been described as a standard and the most efficient strategy in anticancer therapy (33). The present study focused on the effect of CPT on inhibiting proliferation and migration, and it is worth noting that CPT also displays a pro-apoptosis effect in several human cancers including melanoma cells, and colorectal cancer cell lines $(16,17)$. A previous study indicated that CPT can promote apoptosis not only in human lung cancer cells, but also in xeno-grafts and inhibit the growth of tumors in vivo (27). However, the underlying signaling mechanisms of CPT-mediated pro-apoptotic effects are not fully understood. The present results indicated that CPT can reduce IGF-1R phosphorylation and PI3K/Akt activation. It was hypothesized that inhibition of IGF-1R and downstream signaling cascades may also play important roles in the pro-apoptotic effect of CPT. IGF-1 is widely known as a mitogenic and anti-apoptotic factor, and activation of IGF-1R results in both proliferative and antiapoptotic signals in cancer cells (34). Activation of PI3K/Akt signaling cascades have been reported to induce a strong survival signal by arresting cells in the G0/G1 phase of the cell cycle via modulation of mammalian target of rapamycin and regulating cyclin D1 (35). Akt activation can extensively phosphorylate apoptotic effector molecules, such as B cell lymphoma-2, MCL-1, Bim, Bad and many others to promote cell survival $(24,36)$. However, whether CPT can induce apoptosis in lung cancer cells remains unclear and requires further investigation.

In conclusion, the results indicated that CPT not only inhibited the proliferation and migration, but also suppressed the IGF-1 induced proliferation of lung cancer cells. Furthermore, CPT inhibited IGF-1 induced phosphorylation of IGF-1R and Akt, suggesting the anticancer effect of CPT may be attributable to inhibition of the IGF-1R/PI3K/Akt signaling pathway. The data provides evidence that CPT could be developed as a potential therapeutic agent for the treatment of lung cancer.

\section{Acknowledgements}

Not applicable.

\section{Funding}

This work was supported by grants from the Natural Science Foundation of China (grant nos. 81460444, 81660391), the Key Program of the Natural Science Foundation of Jiangxi Province of China (grant no. S2016ZRZDB0105), the Natural Science Foundation of Jiangxi Province of China (grant nos. 20132BBG20010 and 20151BAB205036).

\section{Availability of data and materials}

The datasets used during the present study are available from the corresponding author upon reasonable request.

\section{Authors' contributions}

JZ and BY conceived and designed the study. JZ, GW and LS performed most of the experiments. WY, RW and QZ performed parts of the experiments. GZ performed and analyzed parts of the western blotting data. JZ and BY wrote the manuscript. WY and GZ reviewed and edited the manuscript. All authors read and approved the manuscript and agree to be accountable for all aspects of the research in ensuring that the accuracy or integrity of any part of the work are appropriately investigated and resolved.

\section{Ethics approval and consent to participate}

Not applicable.

\section{Patient consent for publication}

Not applicable.

\section{Competing interests}

The authors declare they have no competing interests. 


\section{References}

1. Siegel RL, Miller KD and Jemal A: Cancer statistics, 2015. CA Cancer J Clin 65: 5-29, 2015.

2. Sakashita S, Sakashita M and Sound Tsao M: Genes and pathology of non-small cell lung carcinoma. Semin Oncol 41 28-39, 2014.

3. Hu P, He J, Liu S, Wang M, Pan B and Zhang W: beta2-adrenergic receptor activation promotes the proliferation of A549 lung cancer cells via the ERK1/2/CREB pathway. Oncol Rep 36 1757-1763, 2016.

4. Youlden DR, Cramb SM and Baade PD: The international epidemiology of lung cancer: Geographical distribution and secular trends. J Thorac Oncol 3: 819-831, 2008.

5. Efferth T, Li PC, Konkimalla VS and Kaina B: From traditional Chinese medicine to rational cancer therapy. Trends Mol Med 13: 353-361, 2007.

6. Tan W, Lu J, Huang M, Li Y, Chen M, Wu G, Gong J, Zhong Z, $\mathrm{Xu} \mathrm{Z}$, Dang Y, et al: Anticancer natural products isolated from chinese medicinal herbs. Chin Med 6: 27, 2011.

7. Singla AK, Garg A and Aggarwal D: Paclitaxel and its formulations. Int J Pharm 235: 179-192, 2002.

8. Wu CY, Cherng JY, Yang YH, Lin CL, Kuan FC, Lin YY, Lin YS, Shu LH, Cheng YC, Liu HT, et al: Danshen improves survival of patients with advanced lung cancer and targeting the relationship between macrophages and lung cancer cells. Oncotarget 8 : 90925-90947, 2017.

9. Chen X, Guo J, Bao J, Lu J and Wang Y: The anticancer properties of Salvia miltiorrhiza Bunge (Danshen): A systematic review. Med Res Rev 34: 768-794, 2014.

10. Shin DS, Kim HN, Shin KD, Yoon YJ, Kim SJ, Han DC and Kwon BM: Cryptotanshinone inhibits constitutive signal transducer and activator of transcription 3 function through blocking the dimerization in DU145 prostate cancer cells. Cancer Res 69: 193-202, 2009.

11. Kim JH, Jeong SJ, Kwon TR, Yun SM, Jung JH, Kim M, Lee HJ, Lee MH, Ko SG, Chen CY, et al: Cryptotanshinone enhances TNF-alpha-induced apoptosis in chronic myeloid leukemia KBM-5 cells. Apoptosis 16: 696-707, 2011.

12. Lu L, Li C, Li D, Wang Y, Zhou C, Shao W, Peng J, You Y, Zhang $X$ and Shen $X$ : Cryptotanshinone inhibits human glioma cell proliferation by suppressing STAT3 signaling. Mol Cell Biochem 381: 273-282, 2013.

13. Park IJ, Yang WK, Nam SH, Hong J, Yang KR, Kim J, Kim SS, Choe W, Kang I and Ha J: Cryptotanshinone induces G1 cell cycle arrest and autophagic cell death by activating the AMP-activated protein kinase signal pathway in HepG2 hepatoma. Apoptosis 19: 615-628, 2014

14. Ge Y, Yang B, Chen Z and Cheng R: Cryptotanshinone suppresses the proliferation and induces the apoptosis of pancreatic cancer cells via the STAT3 signaling pathway. Mol Med Rep 12: 7782 7788,2015

15. Li S, Wang H, Hong L, Liu W, Huang F, Wang J, Wang P, Zhang X and Zhou J: Cryptotanshinone inhibits breast cancer cell growth by suppressing estrogen receptor signaling. Cancer Biol Ther 16: 176-184, 2015 .

16. Li W, Saud SM, Young MR, Colburn NH and Hua B: Cryptotanshinone, a Stat 3 inhibitor, suppresses colorectal cancer proliferation and growth in vitro. Mol Cell Biochem 406: 63-73, 2015.

17. Ye T, Zhu S, Zhu Y, Feng Q, He B, Xiong Y, Zhao L, Zhang Y, Yu L and Yang L: Cryptotanshinone induces melanoma cancer cells apoptosis via ROS-mitochondrial apoptotic pathway and impairs cell migration and invasion. Biomed Pharmacother 82: 319-326, 2016

18. Werner $\mathrm{H}$ and Bruchim I: The insulin-like growth factor-I receptor as an oncogene. Arch Physiol Biochem 115: 58-71, 2009.
19. Resnik JL, Reichart DB, Huey K, Webster NJ and Seely BL: Elevated insulin-like growth factor I receptor autophosphorylation and kinase activity in human breast cancer. Cancer Res 58: $1159-1164,1998$

20. Weber MM, Fottner C, Liu SB, Jung MC, Engelhardt D and Baretton GB: Overexpression of the insulin-like growth factor I receptor in human colon carcinomas. Cancer 95: 2086-2095, 2002.

21. Sharon C, Baranwal S, Patel NJ, Rodriguez-Agudo D, Pandak WM, Majumdar AP, Krystal G and Patel BB: Inhibition of insulin-like growth factor receptor/AKT/mammalian target of rapamycin axis targets colorectal cancer stem cells by attenuating mevalonate-isoprenoid pathway in vitro and in vivo. Oncotarget 6: 15332-15347, 2015.

22. Teng JA, Wu SG, Chen JX, Li Q, Peng F, Zhu Z, Qin J and He ZY: The activation of ERK1/2 and JNK MAPK signaling by insulin/IGF-1 is responsible for the development of colon cancer with type 2 diabetes mellitus. PLoS One 11: e0149822, 2016.

23. Zhou Y, Zeng C, Li X, Wu PL, Yin L, Yu XL, Zhou YF and Xue Q: IGF-I stimulates ERbeta and aromatase expression via IGF1R/PI3K/AKT-mediated transcriptional activation in endometriosis. J Mol Med 94: 887-897, 2016.

24. Hennessy BT, Smith DL, Ram PT, Lu Y and Mills GB: Exploiting the PI3K/AKT pathway for cancer drug discovery. Nat Rev Drug Discov 4: 988-1004, 2005

25. Kim WY, Jin Q, Oh SH, Kim ES, Yang YJ, Lee DH, Feng L, Behrens C, Prudkin L, Miller YE, et al: Elevated epithelial insulin-like growth factor expression is a risk factor for lung cancer development. Cancer Res 69: 7439-7448, 2009.

26. Kim WY, Prudkin L, Feng L, Kim ES, Hennessy B, Lee JS Lee JJ, Glisson B, Lippman SM, Wistuba II, et al: Epidermal growth factor receptor and K-Ras mutations and resistance of lung cancer to insulin-like growth factor 1 receptor tyrosine kinase inhibitors. Cancer 118: 3993-4003, 2012.

27. Chen L, Wang HJ, Xie W, Yao Y, Zhang YS and Wang H: Cryptotanshinone inhibits lung tumorigenesis and induces apoptosis in cancer cells in vitro and in vivo. Mol Med Rep 9: 2447-2452, 2014.

28. Peled N, Wynes MW, Ikeda N, Ohira T, Yoshida K, Qian J, Ilouze M, Brenner R, Kato Y, Mascaux C, et al: Insulin-like growth factor-1 receptor (IGF-1R) as a biomarker for resistance to the tyrosine kinase inhibitor gefitinib in non-small cell lung cancer. Cell Oncol 36: 277-288, 2013.

29. Engelman JA: Targeting PI3K signalling in cancer: Opportunities, challenges and limitations. Nat Rev Cancer 9: 550-562, 2009.

30. Thorpe LM, Yuzugullu H and Zhao JJ: PI3K in cancer: Divergent roles of isoforms, modes of activation and therapeutic targeting. Nat Rev Cancer 15: 7-24, 2015.

31. Sun $\mathrm{CH}$, Chang $\mathrm{YH}$ and Pan CC: Activation of the $\mathrm{PI} 3 \mathrm{~K} / \mathrm{Akt} / \mathrm{mTOR}$ pathway correlates with tumour progression and reduced survival in patients with urothelial carcinoma of the urinary bladder. Histopathology 58: 1054-1063, 2011.

32. Luo J, Manning BD and Cantley LC: Targeting the PI3K-Akt pathway in human cancer: Rationale and promise. Cancer Cell 4: 257-262, 2003

33. Kelly PN and Strasser A: The role of Bcl-2 and its pro-survival relatives in tumourigenesis and cancer therapy. Cell Death Differ 18: 1414-1424, 2011.

34. LeRoith D and Roberts CT Jr: The insulin-like growth factor system and cancer. Cancer Lett 195: 127-137, 2003.

35. De Luca A, Maiello MR, D'Alessio A, Pergameno M and Normanno N: The RAS/RAF/MEK/ERK and the PI3K/AKT signalling pathways: Role in cancer pathogenesis and implications for therapeutic approaches. Expert Opin Ther Targets 16 (Suppl 2): S17-S27, 2012.

36. Datta SR, Dudek H, Tao X, Masters S, Fu H, Gotoh Y and Greenberg ME: Akt phosphorylation of BAD couples survival signals to the cell-intrinsic death machinery. Cell 91: 231-241, 1997. 